\title{
Dynamic control of vestibular dysfunction in elderly patients as a prognostic criterion for changing the cerebral bloodstream
}

\author{
Sergey Hubkin ${ }^{1}$, Algimantas Bubulis ${ }^{2}$, Margarita Dosina ${ }^{3}$, Valentina Rubakhova ${ }^{4}$, \\ Vladimir Minchenya ${ }^{5}$, Svetlana Pashkevich ${ }^{6}$ \\ $1,3,4,6$ The Institute of Physiology of the National Academy of Sciences of Belarus, Minsk, Belarus \\ ${ }^{2}$ Kaunas University of Technology, Kaunas, Lithuania \\ ${ }^{5}$ Belarusian National Technical University, Minsk, Belarus \\ ${ }^{3}$ Corresponding author \\ E-mail: 19oubkin@yandex.ru, ${ }^{2}$ algimantas.bubulis@ktu.lt, ${ }^{3}$ dosina1985@gmail.com, ${ }^{4}$ rubakhova@mail.ru, \\ 5vlad_minch@mail.ru, ${ }^{6}$ skypasht@mail.ru
}

Received 30 October 2020; received in revised form 25 November 2020; accepted 3 December 2020 DOI https://doi.org/10.21595/chs.2020.21820

Check for updates

Copyright $(2020$ Sergey Hubkin, et al. This is an open access article distributed under the Creative Commons Attribution License, which permits unrestricted use, distribution, and reproduction in any medium, provided the original work is properly cited.

\begin{abstract}
In the clinical and electrophysiological laboratory of the Department of Neurology and Neurosurgery BelMAPO on the basis of the neurological departments of the 5th Clinical Hospital in Minsk, an examination and correction of the indications of impaired balance control functions in a group of elderly patients with changing the cerebral bloodstream.
\end{abstract}

Keywords: elderly patients, stabilography, changing the cerebral bloodstream, balance.

\section{Introduction}

The control system for the position of the human body in space is complexly organized and includes many structural elements: the reticular formation of the brain and spinal cord, extrapyramidal and pyramidal, rubrospinal, nigrospinal, cerebellar and vestibular systems [1, 2]. Imbalance in maintaining equilibrium indicates the development of a pathological process, for example, a change in cerebral blood flow, in one of the links of the equilibrium control system $[3,4]$. The vestibular analyzer is the main one in coordinating the activity of involuntary functions, maintaining posture and position in space [5]. In addition to stating the fact of the degree of impairment of the vestibular function $[6,7]$, the level of localization of the pathological process, an important aspect for the clinic is also the assessment of the effectiveness of the treatment $[8,9]$.

The development of new technologies for the neurorehabilitation of patients [10] with balance and mobility dysfunctions closely depends on knowledge of the neurophysiological mechanisms involved in postural stabilization, but they are not well understood. Therefore, there is a need to design the mathematical models which allow investigation of different aspects of motor control. The tests currently used in the clinic allow us to establish the severity of imbalance and coordination of movements, but do not quantify them. The use of non-invasive, high-precision diagnostic equipment is one of the ways to solve the problem. One of these methods includes computer stabilization based on biological feedback, which is one of the most promising approaches in the rehabilitation of patients. One of these techniques is biofeedback-based computer stabilography, which is one of the most promising approaches in the rehabilitation of patients with movement disorders [7,9].

Rehabilitation simulators with biofeedback are currently used to restore the impaired balance function, but today there is no comprehensive analysis of changes in balance when various kinds of signals are applied.

In connection with the above, technologies and devices turn out to be priority ones, with the help of which it is possible to determine the functional state of the systems that control the equilibrium function in a non-invasive and prompt manner. As a result of a complex of therapeutic measures, as well as regular training on the stabilization platform, most patients in general showed 
a stable increase in the effectiveness of balance, as well as an improvement in their condition. It is advisable to pay special attention to the possibilities of computerized control of the productivity of treatment, since stabilometry allows an objective assessment of the effectiveness of various methods of treatment for restoring equilibrium in patients.

The goal of the work to evaluate, using a stabilographic platform, the effectiveness of restoring impaired control of static reflexes in patients after a disturbance in the blood supply to the brain of varying severity.

\section{Materials and methods}

The work was performed on the basis of the Institute of Physiology of the NAS of Belarus. A stabilographic platform was used, created and tested by a senior researcher at the Joint Institute of Mechanical Engineering of the NAS of Belarus V.A. Dubovsky in the process of joint research with the staff of the Institute of Physiology of the NAS of Belarus. The device is patented in the Republic of Belarus as "A device for self-balancing", as well as in the Russian Federation as "A way of assessing a person's ability to purposefully control the movements of the center of gravity of his body". In accordance with the approved examination protocol and regulatory documents, employees of the neurophysiology laboratory of the Institute of Physiology of the NAS of Belarus used the classical method for testing vestibular function in subjects on a stabilographic platform with open and closed eyes.

Analysis of the stabilogram included the effectiveness of maintaining equilibrium; Es + forward sagittal; Es- - sagittal back; Ef + - frontally forward; Ef- - frontally back.

At the initial stage, the main complaints of patients who paid attention to the preparation for the tests were recorded: increased and chronic resistance, emotional lability, drowsiness, meteorological dependence, headaches, and feeling particular complaints of explicit and vertical movement.

The study was carried out by recording the position, deviation and other characteristics of the projection of the common center of gravity on the support plane. These data were recorded digitally using a computer. Observations were carried out with the permission of the subjects. Stay on the platform ranged from 60 seconds to 3-4 minutes and depended on the patient's condition, gender and age, stage of the disease, subjective attitude to the study. The first three to four installation tests were performed without registration in order to educate the patient to new conditions (being on a stabilographic platform). The stability of patients on the platform was expressed in accordance with international criteria in the calculated indicators (RP), which ranged from 0 to $1 \mathrm{E}$ units (1E is the best indicator). The dynamics of RP allowed us to quantify the effectiveness of the therapy. The efficiency of the equilibrium function was estimated by the total deviation of the platform relative to the plane perpendicular to the lines of force of the gravitational field. Used static motor-cognitive tests with biological feedback on the support reaction. The target is the environment, it changes color from red to green. Red is an equilibrium shift, green is a stable position. The sound signal is amplified when the center of gravity is displaced and calms down upon reaching a stable equilibrium.

Systematic training sessions in order to control the nature of the implementation of static and statokinetic reflexes were performed at least two to three times a week.

Group 1 was 34 patients with cerebral blood flow disorders and Group 2 was 22 healthy individuals. Testees were examined using a computerized stabilographic platform by recording the position, deviation and other characteristics of the projection of the general center of gravity on the support plane. We analyzed the process of maintaining a vertical posture by a person, which is affected by the functioning of many body systems (vestibular, visual, proprioceptive, musculoskeletal and higher parts of the central nervous system).

During the experiment, 5 training sessions were conducted on the simulator with the presentation of visual, acoustic, mixed or lack of stimuli. Classes on the simulator were also supplemented by the test " 10 words" by Luria [6]. This test allows you to assess the state of 
memory, fatigue, activity of attention. The number of words retained and reproduced an hour later testified to the nature of the formation of long-term memory.

All observations were carried out with the written permission of the subjects.

\section{Results}

The control group was represented by 42 healthy volunteers from among the cadets of the Department of Neurology of the Belarusian Academy of Postgraduate Education, students of the Belarusian State Medical University and employees of the 5th Clinical Hospital in Minsk. The average age of the examined individuals was 36.7 years (22-63 years). There were 18 men and 24 women. The calculated indicators (RP) of the effectiveness of maintaining equilibrium at the first stage in the control group averaged from 0.1 to 0.74 , and at the end of the examination after five to seven samples, from 0.7 to 0.95 . All examined patients observed an increase in RP with an increase in the number of attempts. Such a pattern was noted in other studies. Thus, RP approached the optimum after short-term training in healthy individuals. So, within one day, 5 measurements of the indicators of the effectiveness of maintaining equilibrium in the subject were performed for 22 years with an interval of 10 minutes. The initially high $(0.77)$ performance indicator for visual stimulus after three workouts rose to 0.96 , for acoustic - from 0,85 to 0.89 . It should be noted the relationship of the age of the examined and the level of the calculated indicator. In persons from 22 to 40 years of age, the values of RP were higher than the initial one, and even more so, after the completion of several sessions. In subjects aged 40 to 63 years, this indicator was $0.2-0.3$ lower. Patients adapted to visual stimuli more easily than to verbal ones.

The specific results of the dynamics of RP in the process of one or more sessions in actually healthy individuals are presented in Fig. 1.

Thus, the stability of being on a stabilographic platform in healthy individuals after short-term training was approaching the optimum. In healthy volunteers, during the first training process, RP ranged from 0.105 to 0.424 and after 5 trainings increased to $0.789-0.896(p<0.05)$.

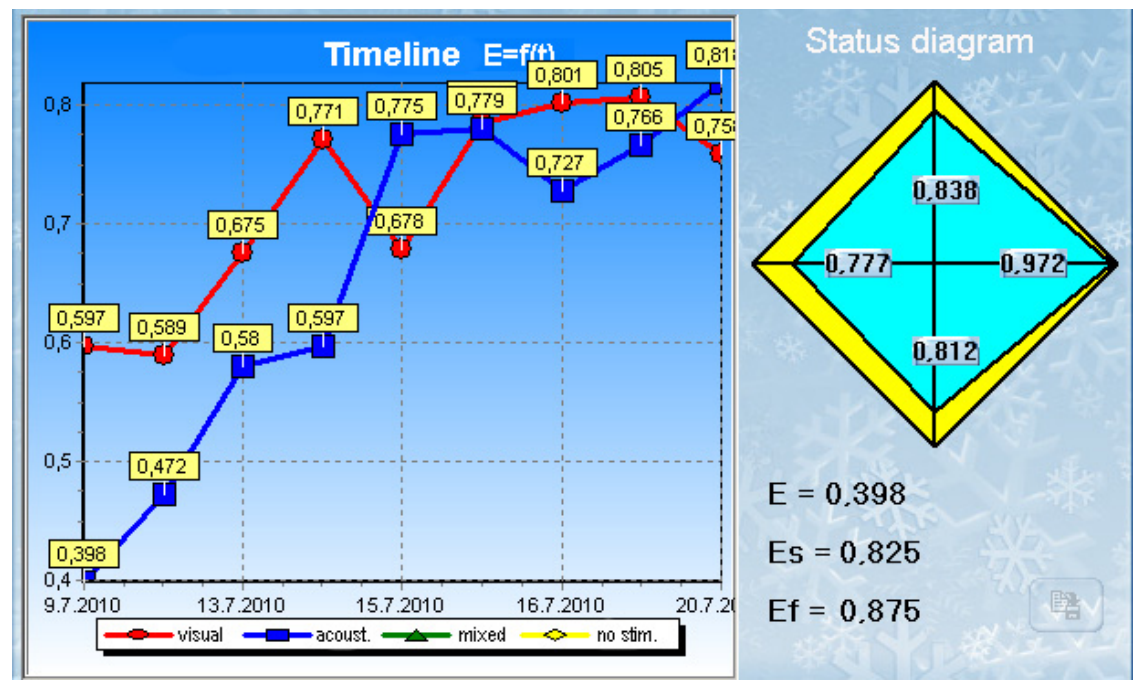

Fig. 1. The variation of indicators of the effectiveness of maintaining equilibrium in the cohort of males born in 1967. The ordinate axis is an indicator of changes in the efficiency of maintaining equilibrium, the abscissa axis is the measurement date.

The circles marked the visual stimulus, the squares marked the sound

Both individuals from the group of healthy volunteers and the patient with changing the cerebral bloodstream were additionally tested for memorizing words. This test allows you to assess the state of memory, fatigue, activity of attention. The number of words retained and 
reproduced an hour later testified to the nature of the formation of long-term memory. After 5 trainings, the processes of formation of long-term memory in patients without disturbance of cerebral circulation improved (from $5 \pm 2$ words to $7 \pm 1$ ) and were accompanied by an increase in the efficiency of controlling the equilibrium function on a stabilographic platform (Fig. 2).

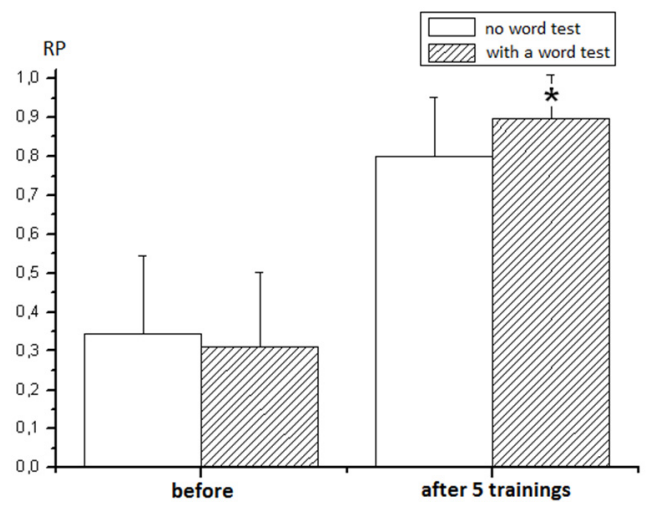

Fig. 2. Dynamics of the effectiveness control of the equilibrium function in the calculated indices (RP) in volunteers before and after 5 trainings on the simulator of motor coordination of a group without a word test and a group with an additional presentation of the " 10 words" test by Luria [6]. * $p<0.05$

The group of examined patients was 38 people aged 48 to 83 years (average age 67.2 years). Females -17 , males -21 , in which a change in cerebral blood flow was recorded.

There is training on a stabilographic platform, carried out for even one day with an interval of 10-20 min, revealed a positive dynamics of equilibrium control in a number of patients with impaired cerebral hemodynamics (Figure 3 ). There is virtually no positive increase in equilibrium control in individuals with complicated encephalopathy, brain cysts and extensive hemorrhages in the process of examining different patients on a stabilographic platform.

Thus, as a result of the complex of therapeutic measures (therapy according to the scheme of vascular preparations, the use of neuroprotectors, anticoagulants, antihypertensive drugs) in combination with stabilography, the function of maintaining equilibrium is normalized. In this case, the positive dynamics of maintaining equilibrium can be expressed quantitatively, using calculated indicators.

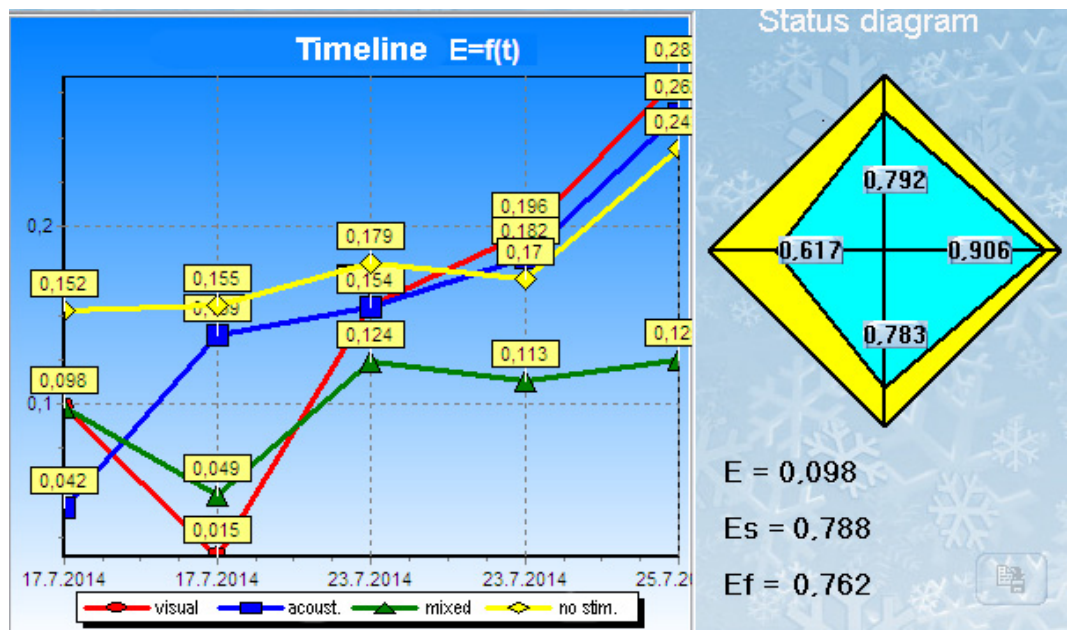

Fig. 3. A graph of changes in the calculated indicators of the effectiveness of maintaining equilibrium in a patient, reflecting the effectiveness of rehabilitation procedures. The circles indicate the visual stimulus, the squares the sonic, the triangles the mixed, the diamonds without the stimulus 
In volunteers from among patients with cerebrovascular accidents, the correction of static reflexes was carried out in dynamics for 1 year. At all during the first training process, RPs ranged from 0.042 to 0.152 and after 5 trainings increased to $0.12-0.28(p<0.05)$. But in some patients with this pathology, against the background of an increase in the calculated indicator of the effectiveness of maintaining equilibrium as a result of training, it periodically decreases. An explanation of what happened can be not only the poor health of the patients examined during a specific period of the disease, which patients often associate with insomnia, fatigue, and intravenous infusion of drugs. The analysis shows that the cause is the structural and functional features and dynamics of the pathological process in the brain.

As in healthy volunteers, in patients after ischemic or hemorrhagic strokes, the memory function was monitored in the process of assessing the equilibrium function.

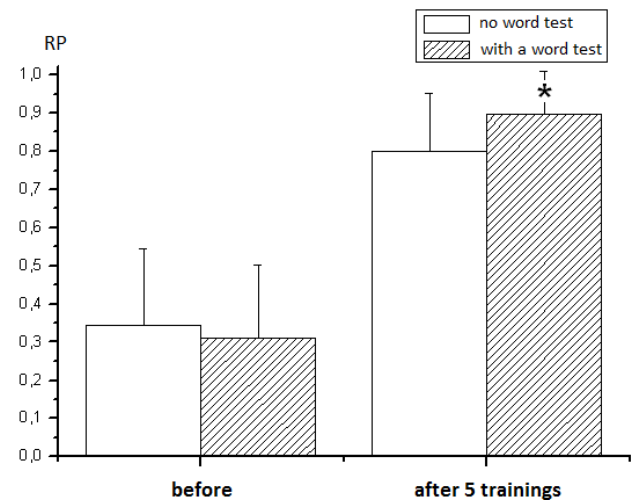

Fig. 4. Dynamics of monitoring the effectiveness of the equilibrium function by calculated indices (RP) in patients with cerebrovascular accidents before and after 5 workouts on the group movement coordination simulator without a word test and a group with an additional presentation of the " 10 words" test by Luria [6]. * $p<0.05$

After 5 trainings, the processes of formation of long-term memory in the group of patients with ischemic and hemorrhagic strokes tended to improve (from $3 \pm 2$ words to $4 \pm 1$ ) and were accompanied by an increase in the efficiency of controlling the equilibrium function on a stabilographic platform (Fig. 4). It should be noted that compared with healthy volunteers, RPs in this experimental group were significantly lower.

\section{Conclusions}

As a result of the complex of therapeutic measures (basic therapy with vascular preparations, neuroprotectors, anticoagulants, antihypertensive agents, hyperbaric oxygenation) and training samples, as a rule, the calculated indicator is normalized. In the majority of the examined, after conducting sessions on the device for self-balancing, improvement was noted not only in physical activity, but also in general condition, confidence in recovery. It is possible to train the patient for a relatively short period to maintain balance and vertical posture on a stabilographic platform, which is a real factor aimed at preventing injuries in patients. In the case of prolonged persistence of dysfunctional manifestations, despite all types of therapy, it is advisable to recommend a change in the treatment regimen or an additional examination of the patient.

\section{Acknowledgements}

This analysis was funded by state research program "Convergence-2020". 


\section{References}

[1] Cardin V., Smith F. T. Parallel motion signals to the medial and lateral motion areas V6 and MT+ Cereb. Cortex, Vol. 20, Issue 8, 2010, p. 1964-1973.

[2] Fujimoto C., Murofushi T., Chihara Y., et al. Effects of unilateral dysfunction of the inferior vestibular nerve system on postural stability. Clinical Neurophysiology, Vol. 121, Issue 8, 2010, p. $1279-1284$.

[3] Puszczalowska-Lizis E., Bujas P., Omorczyk J. Postural stability in women in the eighth and ninth decades of life. Acta of Bioengineering and Biomechanics, Vol. 18, Issue 3, 2016, p. 115-121.

[4] Rosker J., Sarabon N. Kinaesthesia and methods for its assessment. Sport Science Review, Vol. 19, 2010, p. 165-208.

[5] Wouwe N. C., Ridderinkhof K. R., Band G. P., et al. Dose dependent dopaminergic modulation of reward-based learning in Parkinson's disease. Neuropsychologia, Vol. 50, Issue 5, 2012, p. 583-591.

[6] Yamaguchi T., Wang H. L., Li X., et al. Mesocorticolimbic glutamatergic pathway. Journal of Neuroscience, Vol. 31, Issue 23, 2011, p. 8476-8490.

[7] Norre M. E. Posturography: head stabilisation compared with platform recording. Application in vestibular disorders. Acta Oto-Laryngologica, Vol. 520, Issue 2, 1995, p. 434-436.

[8] Redgrave P., Gurney K., Reynolds J. What is reinforced by phasic dopamine signals? Brain Research Reviews, Vol. 58, Issue 2, 2008, p. 322-339.

[9] Lin C. C., Crago P. E. Structural model of the muscle spindle. Annals of Biomedical Engineering, Vol. 30, 2002, p. 68-83.

[10] Sarabon N. Development of software for comprehensive analyses of force plate measurements: technical note. Kinesiology, Vol. 43, 2011, p. 204-212.

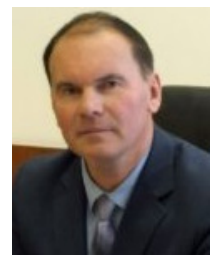

Sergey Hubkin received Ph.D. degree in medical science from Belarusian Medical State University, Minsk, Belarus, in 2008. Now he is Director of the Institute of Physiology of the National Academy of Sciences of Belarus. Professor Sergey Gubkin has been involved in a number of international projects in the field of frequency-modulated angioplasty, GPS cardiomonitor physical condition, Cor-box for delayed heart transplantation, Smart-stent implantable module: Chip-stents - probes for remote monitoring of intravascular pressure, glycemia, coagulation parameters, biostents with a monolayer of autologous endothelial cells, devices for intracardiac distant systemic thrombolysis - implantable cardioverter defibrillators with microinfusate, angioplastic correction of mitral regurgitation, wireless cardiac resynchronization devices, pump modules for pericardial correction of dilatation of the ventricles of the heart.

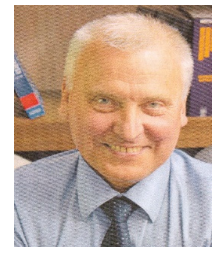

Algimantas Bubulis received Ph.D. degree in 1979. Since 2002 is a Chief researcher and a Habilitated Doctor at Kaunas University of Technology, Kaunas, Lithuania. His main research interests include mechatronics systems, measurement engineering, new technologies in biology and medicine.

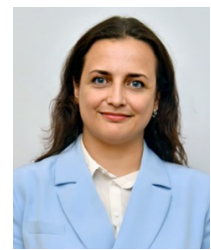

Margarita Dosina received Ph.D. degree in biological science from Institute of Physiology of the National Academy of Sciences of Belarus, Minsk, Belarus, in 2015. Now she is Scientific Secretary Institute of Physiology National Academy of Sciences of Belarus. Her current research interests include pathophysiology, new technologies in biology and medicine. 


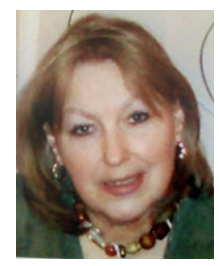

Valentina Rubakhova received Ph.D. degree in biological science from Institute of Physiology of the National Academy of Sciences of Belarus, Minsk, Belarus, in 1986. Now she is Leading Researcher Laboratory of Neurophysiology Institute of Physiology National Academy of Sciences of Belarus. Her current research interests include vestibular dysfunction in elderly patients.

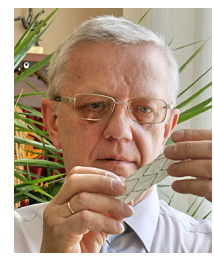

Vladimir Minchenya received Ph.D. degree in technical science from Belarusian Polytechnic Institute, Minsk, Belarus, in 1987. Now he is Professor of the Department of Design and Production of Instruments, Belarusian National Technical University. His current research interests include high-frequency vibrations in engineering and medicine.

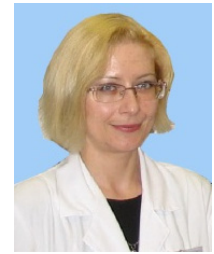

Svetlana Pashkevich received Ph.D. degree in biological science from Institute of Physiology of the National Academy of Sciences of Belarus, Minsk, Belarus, in 2003. Now she is head of laboratory Neurophysiology Institute of Physiology National Academy of Sciences of Belarus. Her current research interests include pathophysiology, experimental neurophysiology. 